$52 \mid 2014$

Grammaire et enseignement du français langue

étrangère et seconde - Permanences et ruptures du

XVIe au milieu du XXe siècle (II)

\title{
L'enseignement/apprentissage de la grammaire dans les manuels de FLE en Turquie (1931-1949)
}

\section{Erdogan Kartal}

\section{OpenEdition}

Journals

Édition électronique

URL : https://journals.openedition.org/dhfles/3589

DOI : $10.4000 /$ dhfles.3589

ISSN : 2221-4038

Éditeur

Société Internationale pour l'Histoire du Français Langue Étrangère ou Seconde

Édition imprimée

Date de publication : 1 juin 2014

Pagination : 91-108

ISSN : 0992-7654

Référence électronique

Erdogan Kartal, « L'enseignement/apprentissage de la grammaire dans les manuels de FLE en Turquie (1931-1949) », Documents pour l'histoire du français langue étrangère ou seconde [En ligne], 52 | 2014, mis en ligne le 06 septembre 2017, consulté le 28 mai 2021. URL : http://journals.openedition.org/ dhfles/3589; DOI : https://doi.org/10.4000/dhfles.3589

Ce document a été généré automatiquement le 28 mai 2021.

(c) SIHFLES 


\title{
L'enseignement/apprentissage de la grammaire dans les manuels de FLE en Turquie (1931-1949)
}

\author{
Erdogan Kartal
}

1 L'histoire des échanges diplomatiques, interculturels et commerciaux franco-turcs remonte au début du XVI e siècle. Malgré de brèves ruptures dans le passé, les deux pays ne cessent d'avoir des échanges. Avec la fondation de la nouvelle République turque (1923), les relations franco-turques prennent une nouvelle forme et s'améliorent par des conventions mutuelles dans tous les domaines. Et le français, jadis «langue des élites » et des "plumistes » (auteurs), s'introduit comme langue étrangère ou langue d'enseignement dans les établissements scolaires turcs. Il devient en même temps un moyen de s'instruire personnellement pour tous ceux qui ont envie de s'investir dans une culture francophone/ occidentale. Cette évolution va jusqu'à son terme au début des années 50, notamment à la fin de la Deuxième Guerre mondiale. Depuis, en Turquie, le français est en recul rapide et continuel face à l'anglais, comme c'est le cas dans plusieurs autres pays.

2 Dans ce travail, nous nous proposons d'aborder la tradition d'enseignement/ apprentissage de la grammaire en Turquie à travers les manuels de français langue étrangère (désormais FLE) utilisés dans la première moitié $d u X X^{e}$ siècle. Pour ce faire, nous allons étudier les techniques et les procédés d'enseignement/apprentissage de la grammaire dans les manuels scolaires et parascolaires. Nous nous basons sur un corpus constitué à partir des manuels parus dans la première moitié $\mathrm{du} \mathrm{XX}^{\mathrm{e}}$ siècle que nous avons retenus par une «recherche simple» dans le catalogue général de la Bibliothèque nationale de Turquie. En ce qui concerne la démarche méthodologique, nous avons suivi l'approche dite analyse documentaire dans l'étude des manuels en question. 


\section{Corpus étudié}

3 Nous avons essayé de constituer un corpus de travail dans les limites et les objectifs d'un colloque qui traite entièrement de l'enseignement de la grammaire française dans une perspective historique. Pour ce faire, avec une recherche simple, réduite à une période de la première moitié du XXe siècle, par le mot-clé turc « Fransızca » (français) du catalogue général en ligne de la Bibliothèque nationale de Turquie (http:// www.mkutup.gov.tr/), nous sommes parvenu à une centaine d'occurrences. Le posttraitement de ces occurrences nous a permis de prélever une trentaine de documents qui correspondent notamment au concept de manuel de grammaire française ou de FLE. Il nous faut souligner que les trois quarts du relevé se composent de dictionnaires et de guides français-turcs. De plus, les documents/supports pédagogiques et culturels font partie également de la liste. Dans une deuxième consultation sur place du répertoire, nous avons retenu neuf manuels qui pourraient constituer un corpus représentatif dans son ensemble pour notre travail en ce qui concerne les objectifs mentionnés ci-dessus. Rappelons enfin que notre corpus se limite aux manuels publiés en Turquie. Les remarques préalables que nous faisons sur les manuels sont les suivantes :

4 1. Ils sont conçus par des auteurs turcs et publiés entre les années 1931 et 1949 par diverses maisons d'éditions turques à Istanbul.

5 2. Ils s'adressent à un vaste public : scolaires (collégiens), grands adolescents et adultes de niveau débutant.

6 3. Les deux tiers (six) des manuels sont proposés pour un apprentissage autonome et ils se présentent sous des titres assez prétentieux tels que: a) Otamatik grammaire: Fransizca öğrenmek için en kestirme yol (Grammaire automatique : le plus court chemin pour apprendre le français); b) Fransızcayı çabuk ve kolay öğretirim (J'enseigne vite et facilement le français); c) Temel fransızca: Fransızcayı öğretici kitap (Français fondamental: Manuel pouvant/capable d'enseigner le français); d) Fransızca dersler (Method Gayur) : 48 derste kendi kendine fransizca (Cours de français (Méthode Gayur) : Le français en 48 cours en autonomie) ; e) Kendi kendine kolay fransızca (Français facile en apprentissage autonome); f) Muallimsiz üç ayda fransızca (Français en trois mois sans précepteur).

7 4. Deux manuels sont conçus pour l'enseignement collectif dans les écoles turques : 1 ) Fransızca ders kitabı I (Manuel de français I) et 2) Resimli fransızca-türkçe metot Berliç (Méthode Berlitz illustrée français-turc à l'usage des élèves).

8 5. Le dernier manuel du corpus est une grammaire purement consacrée à la conjugaison des verbes et à leur utilisation : Fransizca gramer kitabı II : Fillerin tasrif ve kullanışı (Manuel de grammaire française : Conjugaison et utilisation des verbes).

\section{Démarche méthodologique : analyse documentaire}

9 Dans l'étude des manuels du corpus nous avons procédé à une démarche méthodologique dite analyse documentaire. Cette analyse s'est inscrite dans un processus en trois temps (Vigour 2007 : 1-3) : 
10 1. Dans une première approche, en nous aidant des titres et préfaces ainsi que des formes canoniques des leçons, nous avons essayé de dégager les courants didactiques dans la logique desquels ils ont été conçus.

11 2. Dans une deuxième approche, nous avons décrit et caractérisé les tendances relatives à des techniques et procédés d'enseignement/ apprentissage des éléments grammaticaux sélectionnés tels qu'ils sont observables dans les manuels.

12 3. Dans une troisième approche, nous nous sommes efforcé de commenter les principales tendances mentionnées dans la deuxième phase en nous référant à l'histoire de la didactique des langues et de mettre en évidence la tradition d'enseignement/apprentissage de la grammaire en Turquie à travers les manuels parus dans la première moitié $\mathrm{du} \mathrm{XX}^{\mathrm{e}}$ siècle.

\section{3. État des lieux de la didactique grammaticale dans les manuels}

13 Une première étude des manuels du corpus nous a conduit à les situer d'emblée dans l'histoire de la méthodologie des langues. En effet, cette répartition au fil des approches didactiques des langues, notamment du FLE, a facilité la tâche d'analyse qui porterait sur le statut de l'enseignement/apprentissage de la grammaire dans les manuels. Selon leurs ensembles pédagogiques (titre, préface, leçon, consignes, langue/s utilisée/s forme canonique, etc.), ils s'inscrivent dans la conception méthodologique de deux courants didactiques différents qui dominent conjointement en didactique des langues dans la première moitié du XXe siècle (Bolay-Dilek 2007 : 7). Ce sont : a) la méthodologie traditionnelle et b) la méthodologie directe. Il nous semble intéressant de préciser que, même de nos jours, la méthodologie directe est appelée souvent par le nom de son préconisateur : méthode de Berlitz.

\subsection{Grammaire dans les manuels traditionnels}

C'est cette approche qui marque presque entièrement les manuels du corpus. En effet, les sept manuels parus dans un intervalle de 13 ans (1935-1948) sont issus de ce courant pionnier qui jalonne l'histoire de la didactique des langues. Cette approche ayant aussi d'autres appellations telles grammaire-traduction, classique (Germain 1993: 101) ou bilingue (Besse 1985: 25) domine, dès la fin du XVI ${ }^{e}$ siècle, non seulement l'enseignement/apprentissage des langues classiques mais aussi celles vivantes, surtout en Europe, pendant une bonne partie du XXe siècle (Besse 1985 : 27). Il n'est donc pas surprenant de voir qu'elle soit transmise à des manuels par des concepteurs (auteurs) turcs qui ont eux-mêmes subi les mêmes procédés d'enseignement/apprentissage des langues.

15 Pour l'enseignement/apprentissage de la grammaire dans tous ces manuels, il est à noter avant tout qu'aucune rubrique n'est consacrée particulièrement à la grammaire, surtout à une grammaire "consciente». Loin d'un parcours pédagogique et à la fois linguistique proposé en ce qui concerne les étapes essentielles du déroulement d'une leçon, l'accent est mis plutôt sur la langue comme un tout. Elle est traitée sans aucune distinction avec toutes ses composantes: grammaire, lexique, phonétique, etc. Par ailleurs, les sujets grammaticaux dans les cours et leçons ne sont pas présentés dans 
une progression évolutive et cohérente. Ils sont arbitrairement juxtaposés, pas même du plus simple au plus compliqué. Il arrive que dans une seule unité de trois petites pages, le comparatif (plus grand que... / moins frais que... / aussi large que...), les adverbes: très et trop (cette maison très grande... / ce pain est trop frais), l'impératif à la deuxième personne du pluriel des verbes (laisser / manger / boire / lever / écrire et se lever) et les chiffres (de 30 à 100 par dizaines) soient placés consécutivement comme par exemple dans la sixième leçon du manuel Fransizca dersler (Method Gayur) : 48 derste kendi kendine Fransizca (1935 : 34-37).

En règle générale, les cours débutent par la présentation d'une règle grammaticale, le plus souvent dans un tableau récapitulatif, en langue maternelle à l'aide d'un métalangage: «celui, par exemple, d'une terminologie et d'une description grammaticales " (Galisson et Coste $1976: 206$ ). Voici un tableau récapitulatif (Figure 1), issu du manuel Kendi kendine kolay fransizca (1948:27), qui présente l'adjectif possessif (sixième leçon) en français et les explications métalangagières en turc sur le sujet qui le suivent :

Figure 1

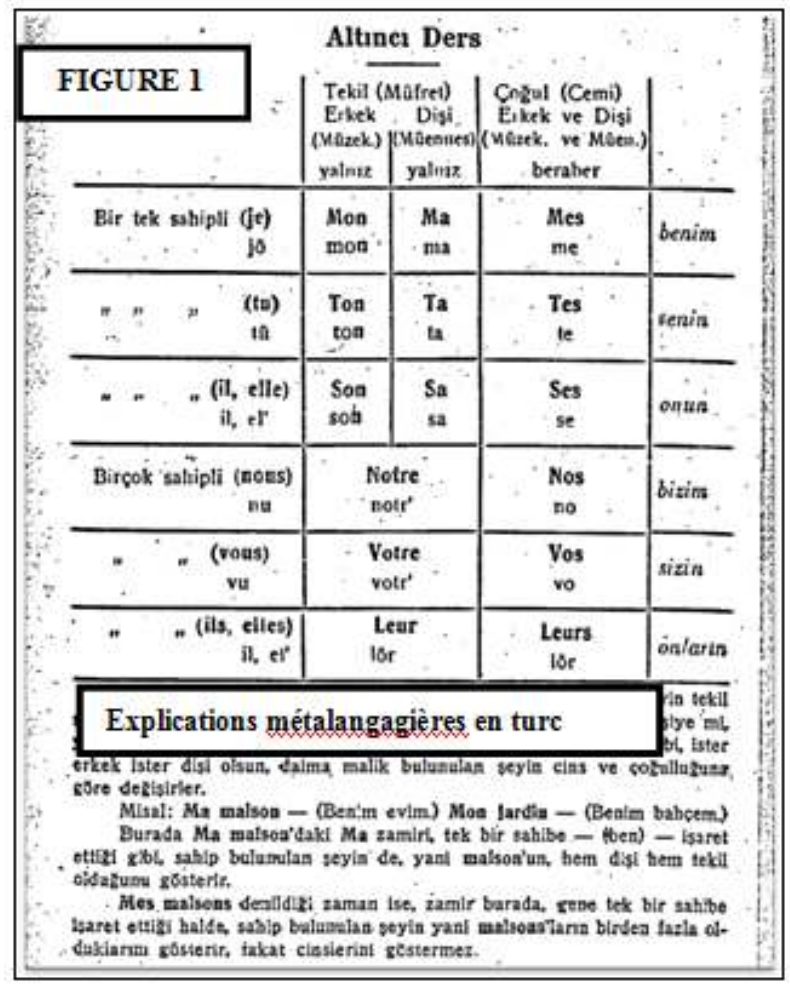

17 Après l'énoncé des règles, elles sont illustrées par des exemples de traduction, ce sont en général des exemples de thème (turc-français). À titre d'exemple nous présentons l'extrait suivant (Figure 2) en guise de rubrique qui s'intitule Misaller (les exemples), tiré du manuel de Method Gagur (1935: 37) : 


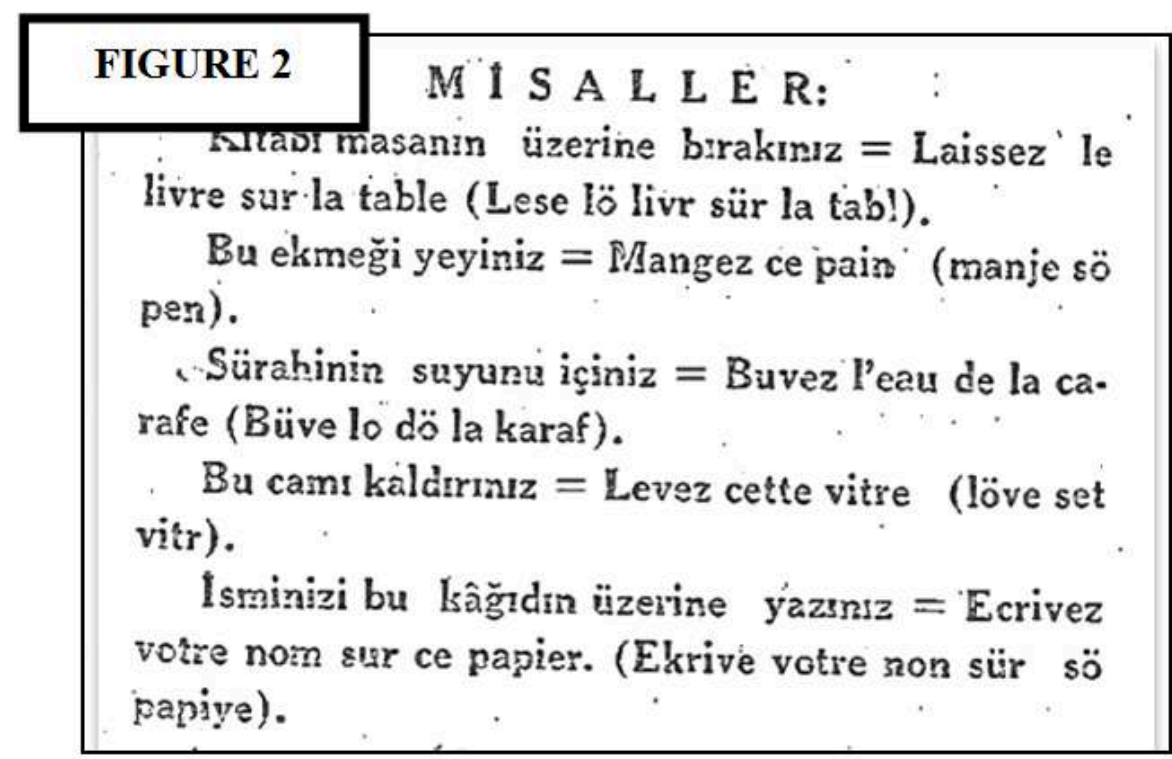

Et les cours ou leçons prennent fin par la vérification des règles déjà abordées à l'aide d'exercices de versions et de thèmes (Tagliante 1999: 31) comme c'est le cas dans presque tous les manuels qui sont conçus dans cette logique. Il nous faut remarquer que, comme le rappellent également Besse et Porquier (1984), les phrases proposées dans tous ces exercices sont souvent artificielles et hors-contextuelles comme dans les exemples de différentes activités de traduction ci-dessous :

19 1. La première activité (Figure 3) İlave talimler (exercices supplémentaires), extraite du manuel Muallimsiz üç ayda Fransizca (1943: 41), consiste en deux exercices (58: version et 59 : thème) par la traduction de phrases courtes et indépendantes.

2. Alors que la deuxième (Figure 4) Yazılı egzersis: Aşağıldaki Türkçe-sualli-cevaph konuşmayı Fransızcaya çevirin (Exercice écrit: Traduisez le dialogue ci-dessus en français) dans le manuel Temel Fransızca (1948: 141), n'est consacrée qu'à la traduction $\mathrm{du}$ turc en français (thème) d'un dialogue simple de 10 questions-réponses (affirmative/ infirmative) :

Figure 3

\begin{tabular}{|c|}
\hline ILÁVE TALIVILER \\
\hline 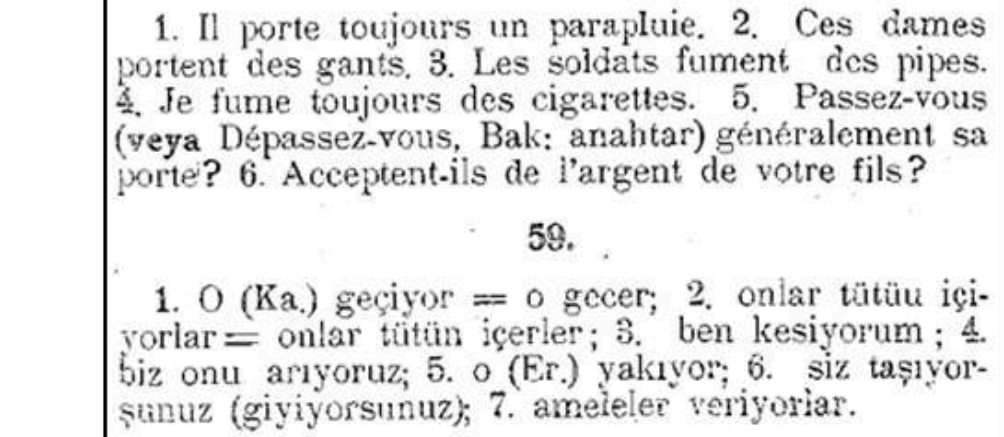 \\
\hline
\end{tabular}


Figure 4

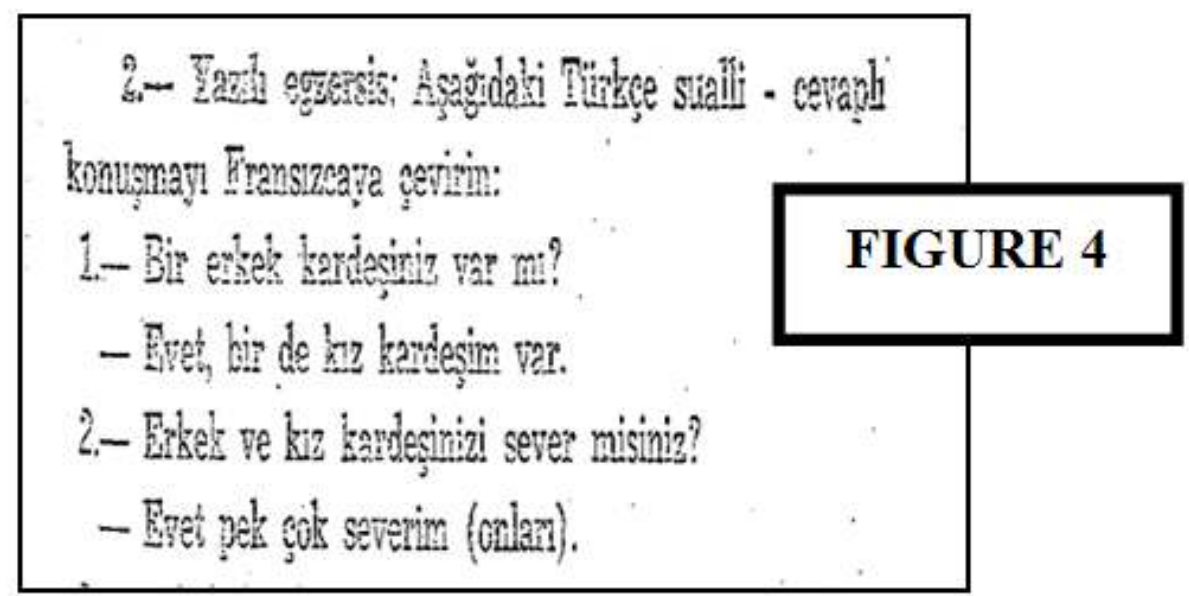

Et finalement, nous avons vu que les exercices de traduction largement répandus dans ces manuels sont systématiquement utilisés comme techniques d'apprentissage de la langue, notamment de la grammaire. Cette dernière est enseignée de façon explicite et déductive (de la règle vers l'application à des cas particuliers), conformément à leur conception de la langue : car d'après Sterne (1983 : 455, dans Germain 1993 : 103), la langue est conçue dans cette approche comme un ensemble de règles et d'exceptions observables dans des phrases ou des textes, susceptibles d'être rapprochées des règles de la langue de départ. À cet effet, les règles sont présentées à l'aide d'un métalangage (Seara 2001) et puis illustrées par des exemples de traduction et viennent ensuite les activités de traduction afin de vérifier si elles ont été assimilées par des élèves.

\subsection{Grammaire dans les manuels directs}

\section{a) Exemple de Fransızca Ders Kitabı I (1949)}

Comme ses auteurs le soulignent dans les pages d'Avertissement qui préfacent le manuel, prévues comme un mode d'emploi pédagogique, ce livre visant un public collégien est spécialement conçu pour l'enseignement collectif dans les écoles turques (1949: 3). L'accent est par ailleurs mis sur le fait qu'il est inscrit dans la logique d'une approche directe et qu'il appartient au professeur de s'engager donc à l'utiliser dans cette perspective didactique afin d'amener ses élèves à la maîtrise effective du français (ibid. : 6). Et il lui est indispensable d'éviter, autant qu'il le peut, la traduction car «la traduction provoque des comparaisons qui deviennent une source constante d'erreurs et d'habitudes défectueuses » (ibid.: 3). C'est par cette idée fondamentale, à l'origine d'un principe particulier, que cette méthodologie émerge et s'oppose aux méthodes traditionnelles. Elle a principalement pour objectif d'amener l'élève à la fois à penser et à s'exprimer directement en langue étrangère, sans avoir recours à la traduction mentale (Puren $1988: 122$ ). Du point de vue de la théorie psychologique, elle est fondée sur la théorie associationniste selon laquelle

le sens renvoie à la forme et vice-versa: la chose (l'objet, l'image, le geste) est

immédiatement associée aux mots et aux sons dans la langue étrangère ; de même, 
l'association directe consent de relier des idées, des concepts abstraits inconnus à

leurs formes écrites et orales par le biais du vocabulaire possédé. (Finotti $2010: 27$ ) grammatical à partir duquel on essaie de découvrir la règle (Puren et al. 1998 : 81), mais cette fois celle-ci est contextualisée à l'aide d'une image illustrative. Par ailleurs, comme le fait remarquer également Besse (1985: 32), les exemples se limitent aux phrases ou formes déjà pratiquées par les élèves afin de leur suggérer visuellement qu'il existe des régularités désinentielles ou des constructions caractéristiques de la langue enseignée, notamment $d u$ français. Ces paradigmes de formes et séries d'exemples sont bien choisis et pourraient permettre aux élèves d'induire la règle, sans que le professeur doive l'expliquer ou la formuler. À titre d'exemple nous proposons cidessous la première page de la sixième leçon (p. 22) où le verbe être est abordé (Figure 5) :

Figure 5

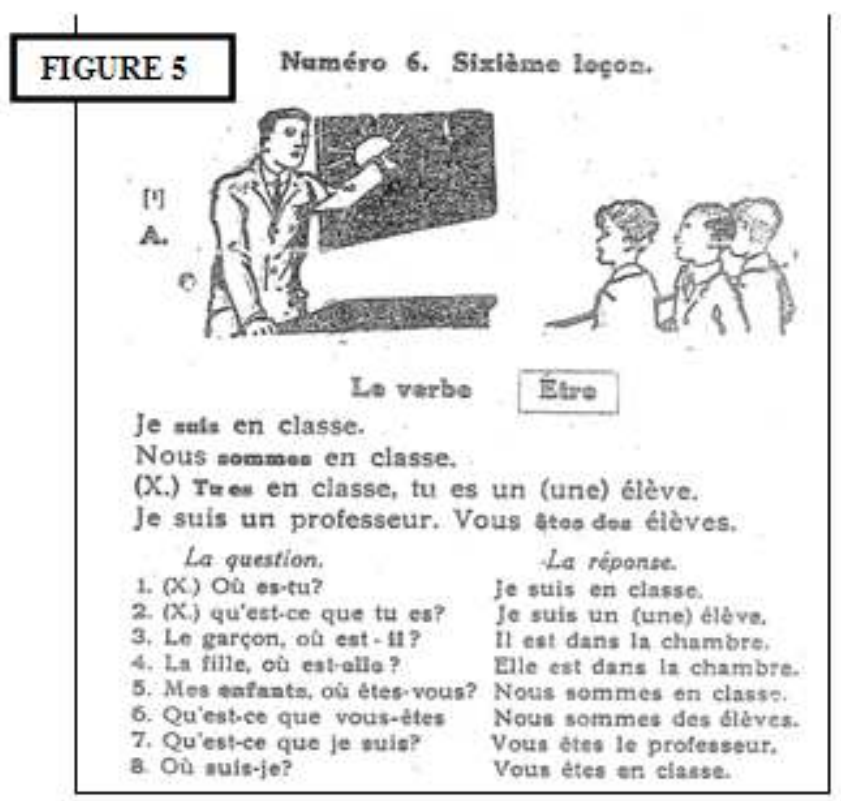

Il en résulte que l'enseignement de la grammaire française dans ce manuel suit une démarche inductive et implicite : allant d'un ensemble de cas particuliers à la règle générale. La présentation des règles se réalise également à partir des exercices de conversation sous la forme de questions-réponses dirigées par l'enseignant, sans passer par l'intermédiaire de la langue maternelle. C'est par ces exercices oraux que, comme le remarquent Germain et Séguin (1995: 19), « il sera possible à l'apprenant de recourir de 
façon spontanée aux formes linguistiques lorsque le besoin se fera sentir ». La compréhension se fait donc de manière intuitive.

Selon Puren (1988: 195), c'est une grammaire relativement simplifiée et graduée par rapport à celle de la méthodologie traditionnelle scolaire, mais elle est toujours normative et construite à partir de la langue écrite littéraire. Il est à noter par ailleurs qu'aucun exercice ou activité, que ce soit à part ou supplémentaire, n'est proposé dans les pages suivantes de la leçon pour la vérification de la règle.

\section{b) Exemple de Resimli Fransızca - Türkçe Metot Berliç}

27 Ce manuel qui date de 1931 constitue le plus ancien document du corpus. Il est conçu également pour un enseignement collectif au niveau secondaire (collège et lycée) pour les élèves turcs (Mektep talebesine mahsus : à l'usage des élèves). Il nous faut rappeler par ailleurs que ce manuel, paru sous la même dénomination Berlitz, n'est qu'une adaptation entière du Premier livre (1889) de la partie française de la fameuse méthode de langue Berlitz (Berlitz 1889) ${ }^{1}$. Cette méthode, élaborée vers la fin du XIX ${ }^{\mathrm{e}}$ siècle aux États-Unis par Maximilian Delphinus Berlitz (1852-1921), enseignant de langue d'origine allemande, fondateur aussi de la première École Berlitz en 1878 à Providence (États-Unis), est considérée comme la promotrice de la méthodologie directe dans l'histoire de la didactique des langues (Germain 1993: 127; Bouquet 1999: 66). De même, selon Reparaz Ruiz (1946: 168), malgré ses quelques inconvénients, cette nouvelle approche « révolutionnaire » marquait à l'époque un progrès considérable par rapport à l'approche traditionnelle qui était purement mnémotechnique et bornée aux seules règles grammaticales (thèmes et versions).

Sa théorie langagière porte sur une optique "naturelle", à savoir qu'elle attribue à chaque habileté une priorité différente conformément à ce qui se passe dans l'acquisition de la langue maternelle (Finotti 2010 : 21). La langue est considérée avant tout comme un phénomène essentiellement oral et la priorité est donc accordée à la prononciation et à la langue parlée dans un objectif d'éduquer l'oreille et d'améliorer l'expression. Ainsi l'apprentissage/acquisition s'effectue-t-il par l'imitation des sons et des phrases calquée sur l'apprentissage/acquisition de la langue maternelle de l'apprenant (Colin 1992: 171).

Pour ce qui est de la grammaire, en tant que réalisation orale liée à la situation, la langue n'est plus un code fait de règles et elle ne coïncide donc pas avec la grammaire. Elle est constituée plutôt de lexique, cependant ceci ne signifie pas qu'elle est une simple juxtaposition de mots mais l'arrangement des phrases introduites dans un contexte. Par conséquent, la langue découle comme une généralisation de faits observés dans les livres et les conversations (Finotti $2010: 21$ ). Ainsi la grammaire est acquise sans efforts dans la conversation. L'extrait ci-dessous (première page de la sixième leçon, p. 21), tiré du manuel, illustre bien cette démarche (Figure 6) : 


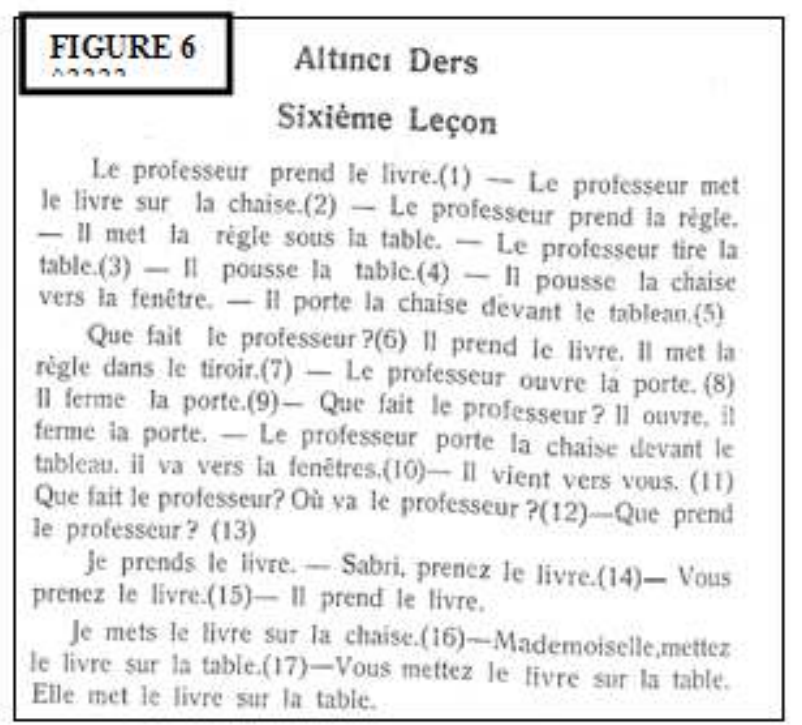

Comme nous le remarquons dans l'extrait, les règles ne sont pas explicitées, par contre il existe une réflexion sur la façon de proposer les notions grammaticales aux apprenants. Il apparaît que la conjugaison au présent de l'indicatif et de l'impératif des verbes prendre-mettre, pousser-tirer-porter, ouvrir-fermer, venir-aller dans un ensemble " antonyme-synonyme », ainsi que l'interrogation avec que, où, est-ce que et l'inversion sont enseignées à l'aide d'un lexique concret appartenant à la sphère de la classe (professeur / livre / chaise / règle / table / fenêtre / tableau / tiroir / porte). En ce qui concerne la pratique de la classe, même si aucune consigne n'est proposée pour la démarche de la leçon, il est évident que c'est le professeur qui décrit ses actions en agissant (premier paragraphe : répliques : 1-6) et les élèves, eux, lui répondent à leur tour comme s'ils étaient dans un dialogue (deuxième paragraphe: 6-13). Avec l'enchaînement répétitif - qu'il soit dialogué ou en monologue - des mêmes faits lexicaux et grammaticaux (les mêmes verbes) conjugués selon les autres pronoms personnels (je / vous / elle) et les sujets tels que Sabri, Mademoiselle Alié (14-33).

Il s'ensuit que, tout comme dans la précédente approche directe, la grammaire est également enseignée de façon inductive et implicite dans ce manuel. Conformément à son approche directe, la notion grammaticale inconnue est présentée en situations monologuées et dialoguées au moyen des exercices systématiques, mais comme le souligne Besse (1987: 195), sans utiliser de métalangage. Il faut souligner que ces situations sont visuellement simulées et contextualisées en salle de classe par les gestes et les actions du professeur car la perception visuelle joue un rôle irremplaçable dans cette méthode, surtout pour un public débutant (Finotti $2010: 21$ ).

Il est à noter que la leçon se termine par une partie d'exercices de traduction en français (thème) (Figure 7, p. 22) qui ne se compose que de phrases de version (en turc) des structures données au début du cours, avec un nombre identique à celles françaises (33 structures). Sachant que cette approche interdit strictement la traduction et le recours à la langue maternelle comme procédé d'enseignement/apprentissage (Soyupek 2004: 16), car selon son précurseur (Berlitz 1898: 31, cité par Finotti 2010 : 26), même dans l'enseignement/apprentissage de la grammaire, l'utilisation de la langue maternelle de l'élève n'a pas de sens, voire est même nocive. En effet, nous 
pensons que cette rubrique avait été prévue et adaptée par son concepteur turc comme un moyen de renforcement des structures apprises pendant les heures de cours, ainsi qu'un instrument d'évaluation pour l'enseignant et d'auto-évaluation pour l'élève même.

Figure 7

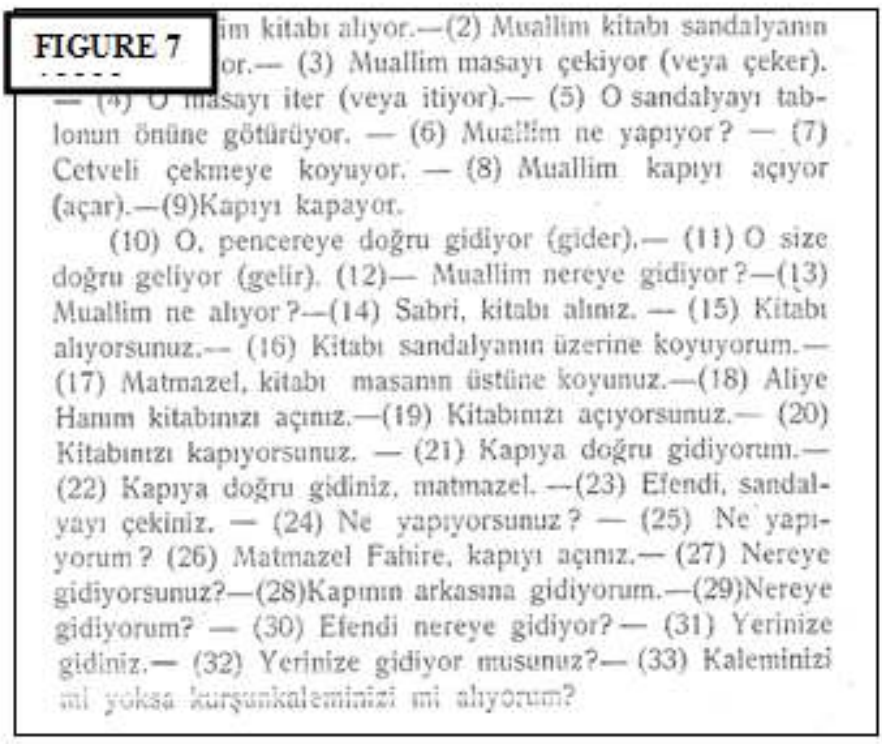
rappelle Puren $(1988: 123)$, il y a eu à l'époque même parmi les promoteurs de cette approche de nombreuses confusions. Certains étaient en fait en faveur du recours à la traduction comme a) méthode d'enseignement linguistique, b) procédé de contrôle de la compréhension linguistique, et c) méthode d'enseignement littéraire dans la didactique des langues, tandis que les autres étaient absolument contre son utilisation dans ce processus, prétendant que la traduction empêchait les élèves de contracter des habitudes linguistiques. Pourtant, Lambert Sauveur (1826-1907), en tant qu'un des grands initiateurs outre-Atlantique de ce courant, ne voyait aucune contradiction dans l'emploi de la traduction comme procédé d'explication lexicale et grammaticale à condition qu'elle intervienne au bon moment (Sauveur $1875: 9$, dans Finotti $2010: 25$ ) : «Nous traduisons dans nos classes. La traduction est indispensable pour achever l'étude de la langue, tout autant que la grammaire, mais comme la grammaire elle doit venir à son heure ». Nous imaginons que c'est dans cette conception que ce manuel a été adapté à l'usage d'un public collégien ou lycéen turc.

\section{Pour conclure}

L'étude des manuels du corpus révèle que tous ces manuels parus dans un contexte local, notamment en Turquie, se meuvent dans la ligne de deux grands courants didactiques, à savoir la méthodologie traditionnelle et la méthodologie directe. Cette oscillation entre ces deux grandes approches qui s'opposent l'une à l'autre découle en fait d'une longue filiation de ruptures, continuités et retours en arrière dans l'histoire de la méthodologie des langues, car comme le souligne Galisson (1980:134), « toutes les

Documents pour l'histoire du français langue étrangère ou seconde, 52 | 2014 
approches dérivent les unes des autres, parce qu'elles s'imitent et parce qu'elles s'opposent ».

Effectivement, le deuxième quart du $\mathrm{XX}^{\mathrm{e}}$ siècle où les manuels étaient en usage (1931-1949), juste avant, pendant et après la Deuxième Guerre mondiale, correspond également à une période mouvementée où les relations et échanges internationaux au niveau social, culturel, diplomatique et commercial deviennent plus denses et plus rapides. Ainsi la communication, notamment l'oralité, devient désormais le but principal dans la didactique des langues vivantes. Dès lors, l'apprentissage devrait passer d'abord par l'audition et la perception, par l'oreille plutôt que par l'œil. C'est pourquoi les manuels conçus dans une telle optique "directe " s'opposant à celle précédente (traditionnelle), voient le jour et s'introduisent surtout dans les cursus scolaires en Europe.

C'est dans ce paysage didactique et historique que, semble-t-il, les deux manuels directs du corpus (Fransızca ders kitabı I et Resimli fransızca-türkçe metot Berliç) sont conçus pour un enseignement collectif dans les établissements turcs, même si la quasi-totalité des autres manuels en usage durant cette période procède encore d'une approche bilingue. Bien que ces derniers dominent le champ didactique français et fassent sentir leurs quelques traits caractéristiques vis-à-vis de ceux qui sont en train de leur succéder, comme par exemple l'utilisation des exercices de traduction dans l'adaptation de méthode Berlitz, nous entendons de plus en plus les bruits de pas des manuels directs, surtout dans le contexte institutionnel.

Notons par ailleurs que ces décennies (1930-50) portent, en Turquie, sur une époque où les réformes entreprises par Mustafa Kemal Atatürk telles que politiques, économiques, sociales, culturelles et éducatives, à savoir unification de l'enseignement en 1924, révolution des signes en 1928 et réforme de l'Université en 1933, ont déjà été mises sur pied. Dans un tel contexte, le français est un cas privilégié en tant que langue étrangère surtout chez les élites et les intellectuels, favorisée par une forte demande de la part des citoyens de la jeune République de Turquie. Ainsi nous constatons qu'un nombre de manuels parus sous différents noms sloganistes/propagandistes, même s'ils s'inscrivent dans une logique traditionnelle, sont conçus par les éditions turques pour un apprentissage autonome, tout en tenant compte de la demande, avec un but commercial bien entendu.

Pour ce qui est de l'analyse des techniques et procédés de la didactisation des faits grammaticaux dans tous ces manuels, nous observons naturellement deux procédés différents: a) ce sont les manuels directs qui cherchent à suivre une démarche inductive et implicite à l'aide d'exercices de conversation dont le procédé d'acquisition/apprentissage porte en général sur la répétition et b) conformément à leur optique langagière, notamment ceux qui reviennent d'une succession traditionnelle, il va de soi qu'ils procèdent d'une démarche explicite, à la fois métalangagière et déductive qui part de la règle vers l'application à des cas particuliers à travers des exercices de traduction bilingues.

39 En somme, il faut souligner que les manuels, tant du point de vue du parcours pédagogique que du contenu linguistique, ne sont pas bien conçus. Par contre n'oublions pas qu'à cette époque, le monde de la didactique des langues, notamment celui du FLE, est loin du véritable concept de manuel de langue, et que les exercices structuraux n'ont pas cours pour la systématisation des acquis. On devra attendre le milieu des années 50 pour voir l'apparition du fameux « Mauger bleu » (Cours de langue 
et de civilisation française, 1953) qui va circuler dans le monde entier. Et c'est dans sa suite que le champ va s'industrialiser.

\section{BIBLIOGRAPHIE}

\section{Corpus de manuels}

AKÇi̇, Mithat (1946). Kendi kendine kolay fransızca [Français facile en apprentissage autonome]. Istanbul : Mithat Akçit.

AYEL, Edip (1943). Fransızca gramer kitabı II : Fillerin tasrif ve kullanışı [Manuel de grammaire française : Conjugaison et utilisation des verbes]. Istanbul : Edip Ayel.

BLEDA, M. Gayur (1935). Fransizca dersler (Metot Gayur) : 48 derste kendi kendine fransizca [Cours de français (Méthode Gayur) : Le français en 48 cours en autonomie]. Istanbul : Vakit Matbaası.

GÜÇER, Behçet, COUDERT, Victor, BERGEAUD, Camille \& ÜN, İ. Hamit (1949). Fransızca ders kitabı I [Manuel de français I]. Istanbul : Milli Eğitim Basımevi.

MÜNÜR, Muallim (1935). Fransızcayı çabuk ve kolay öğretirim [J'enseigne vite et facilement le français]. Istanbul : Necmistikbal Matbaası.

OKCUGIL, K. M. Vasıf (1943). Muallimsiz üç ayda fransızca [Français en trois mois sans précepteur]. Istanbul : M. Sadık Hitay.

RIFKI, Ragıp (1931). Resimli fransızca-türkçe metot Berliç [Méthode Berlitz illustrée français-turc à l'usage des élèves]. Istanbul : şirketi Mürettibiye Matbaası.

TEMIZER, Abbas (1948). Temel fransızca : Fransızcayı öğretici kitap [Français fondamental : Manuel pouvant/capable d'enseigner le français]. Istanbul : Ahmet Sait Kitabevi.

TUNCEL, Mitat. (1936). Otamatik grammaire : Fransızca öğrenmek için en kestirme yol [Grammaire automatique : Le plus court chemin pour apprendre le français]. Istanbul : Reklam Basımevi.

Études

BESSE, Henri (1985). Méthodes et pratiques des manuels de langue. Paris : CREDIF.

BESSE, Henri (1987). « Documents authentiques et enseignement/ apprentissage de la grammaire d'une langue étrangère ». In Jacques Cortès (dir.). Une introduction à la recherche scientifique en didactique des langues. Paris : CREDIF, 183-214.

BESSE, Henri \& PORQUIER, Rémy (1984). Grammaires et didactique des langues. Paris : HatierCREDIF.

BOLAY-DILEK, Feyza (2007). La traduction pédagogique dans la traduction de presse (Mémoire de maîtrise non publié). Ankara : Université Gazi, Institut des sciences pédagogiques.

BOUQUET, Simon (1999). « De la méthode directe aux investigations philosophiques de Wittgenstein : Savoirs et transferts de savoirs ». Langage et société, 86, 41-77.

COLIN, Mariella (1992). « Pour une histoire de l'enseignement du français en Italie » (Compte rendu). Histoire de l'éducation, 54, 168-171. 
FINOTTI, Irene (2010). Lambert Sauveur à l'ombre de Maximilian Berlitz: Les débuts de la méthode directe aux États-Unis. Bologne : CLUEB.

GALISSON, Robert (1980). D'hier à aujourd'hui, la didactique des langues étrangères : du structuralisme au fonctionnalisme. Paris : Clé International.

GALISSON, Robert \& COSTE, Daniel (1976). Dictionnaire de didactique des langues. Paris : Hachette.

GERMAIN, Claude (1993). Évolution de l'enseignement des langues : 5000 ans d'histoire. Paris : Clé International.

GERMAIN, Claude \& SEGUIN, Hubert (1995). Le point sur... la grammaire en didactique des langues. Anjou (Québec) : Les éditions CEC.

GÜLMEZ, Gülnihal (1993). Méthodes d'enseignement du français langue étrangère. Eskişehir : Anadolu Üniversitesi Yayınları.

PUREN, Christian, BERTOCCHINI, Paola \& COSTANZO, Edvige (1998). Se former en didactique des langues. Paris : Ellipses.

PUREN, Christian (1988). Histoire des méthodologies de l'enseignement des langues. Paris : Nathan, Clé International.

REPARAZ RUIZ, Gonzalo de (1946). «Les études hispaniques aux États-Unis jusqu'en 1939 (suite et fin) ». Bulletin Hispanique, 48(2), 147-169.

SEARA RODRÍGUEZ, Ana (2001). «L'évolution des méthodologies dans l'enseignement du français langue étrangère depuis la méthodologie traditionnelle jusqu'à nos jours ». Revista de Humanidades [En ligne], 1, Article 8, consulté le 20 mai 2012. URL : http://www.uned.es/catudela/revista/index_publi.htm.

SOYUPEK, Hasan (2004). İkinci Meşrutiyet'ten günümüze Türkiye'de arapça öğretimi [De la deuxième monarchie constitutionnelle jusqu'à nos jours : l'enseignement de l'arabe en Turquie] (Thèse de doctorat non publiée). Isparta : Université Süleyman Demirel, Institut des Sciences sociales, Département des Sciences islamiques de base.

TAGLIANTE, Christine (1994). La classe de langue. Paris : Clé International.

VIGOUR, Cécile (2007). La méthode du commentaire de documents (Fiche méthodologique) [En ligne], consulté le 23 mai 2012. URL : http://www.melissa.ens-cachan.fr/IMG/pdf/Commenter. Documents.pdf.

\section{NOTES}

1. Berlitz Maximilian D. (1889), Méthode Berlitz. Pour l'enseignement des langues modernes. Partie française. Premier livre, Berlin : S. Cronbach. 


\section{RÉSUMÉS}

Dans cette étude, en nous basant sur un corpus de manuels scolaires et parascolaires de français langue étrangère (FLE), parus dans les premières décennies de la jeune République turque (1931-1949), manuels qui, à noter au passage, ne sont pas nombreux et variés, nous cherchons à en dégager la tradition d'enseignement/apprentissage de la grammaire française dans ce contexte historique. C'est l'approche dite analyse documentaire qui intervient dans l'étude des manuels en question.

Basing on a corpus of curricular and extracurricular textbooks of French as a Foreign Language published in the first decades of the young Turkish Republic (1931-1949), this study aims to identify the tradition of teaching / learning of French grammar in a historical context. In order to do that, the books in question were analyzed with documentary analysis as a research tool.

\section{INDEX}

Keywords : grammar teaching, textbooks of French as a foreign language, Turkey, traditional methods, direct method, Berlitz method, documentary analysis

Mots-clés : didactique grammaticale, manuel de français langue étrangère, Turquie, méthode traditionnelle, méthode directe, méthode Berlitz, analyse documentaire

\section{AUTEUR}

\section{ERDOGAN KARTAL}

Université Uludag, Bursa, Turquie

ekartal@uludag.edu.tr 